AperTO - Archivio Istituzionale Open Access dell'Università di Torino

Radioembolization vs sorafenib in locally advanced hepatocellular carcinoma with portal vein tumor thrombosis: A propensity score and Bayesian analysis

This is a pre print version of the following article:

Original Citation:

Availability:

This version is available http://hdl.handle.net/2318/1807904

since 2021-09-30T16:56:18Z

Published version:

DOI:10.1111/1751-2980.13030

Terms of use:

Open Access

Anyone can freely access the full text of works made available as "Open Access". Works made available under a Creative Commons license can be used according to the terms and conditions of said license. Use of all other works requires consent of the right holder (author or publisher) if not exempted from copyright protection by the applicable law. 


\title{
Radioembolization vs sorafenib in locally advanced hepatocellular carcinoma with portal vein tumor thrombosis: A propensity score and Bayesian analysis
}

Carolina Martelletti ${ }^{1,8}$, Andrea Ricotti ${ }^{5,6}$, Marcantonio Gesualdo ${ }^{8}$, Patrizia Carucci $^{1}$, Silvia Gaia ${ }^{1}$, Michela Emma Burlone ${ }^{2}$, Stefano Okolicsanyi ${ }^{3}$, Alberto Mattalia ${ }^{4}$, Mario Pirisi ${ }^{2}$, Paola Berchialla ${ }^{7}$, Marco Tabone ${ }^{8}$.

${ }^{1}$ Division of Gastroenterology, Città della Salute e della Scienza di Torino, Turin, Italy

${ }^{2}$ Internal Medicine Division, Maggiore della Carità Hospital, Novara, Italy

${ }^{3}$ Division of Gastroenterology, Regional Hospital of Aosta Valley, Aosta, Italy

${ }^{4}$ Division of Gastroenterology, Santa Croce e Carle General Hospital, Cuneo, Italy

${ }^{5}$ Medical Direction of Hospital, Mauriziano Hospital, Torino, Italy

${ }^{6}$ Department of Public Health and Pediatric, University of Torino, Torino, Italy

${ }^{7}$ Department of Clinical and Biological science, University of Torino, Torino, Italy

${ }^{8}$ Division of gastroenterology, Mauriziano Hospital, Turin, Italy

\begin{abstract}
Background \& Aims: Sorafenib is the recommended treatment for patients with advanced hepatocellular carcinoma (HCC). However, radioembolization (TARE) of HCC provides encouraging results both in terms of survival and capability to rescue unresectable tumors to surgery. This study evaluates the outcome reached with TARE and sorafenib in patients with HCC and intrahepatic portal vein tumor thrombosis (PVTT).

Methods: Sixty-five patients with HCC and intra-hepatic PVTT treated in five Italian hospitals between 2012 and 2018 were included in the analysis. Exclusion criteria were any previous treatment, extension of PVTT to the main portal tract and extrahepatic involvement. Propensity score and Bayesian analysis were performed.
\end{abstract}

Results: Forty-one patients received TARE and 24 sorafenib. Eleven patients were rescued to curative-intent surgery ( 3 liver transplant and 8 hepatectomy), 10 with TARE and 1 with sorafenib; TARE was significantly more effective than sorafenib in downstaging patients to surgery providing a mean survival of 54 months. In the 54 patients without downstaging after treatment, 31 treated with TARE and 23 with sorafenib, median survival was 20 months and 9 months respectively $(\mathrm{p}=0.001)$, with different 1, 2- and 3- years OS rates $(64 \%, 43 \%$ and $37 \%$ vs 39\%, $13 \%$ and $0 \%)$. Both propensity 
score and Bayesian model averaging confirmed an improvement in overall survival in TARE group compared to sorafenib treatment.

Conclusions: TARE was more effective than sorafenib in downstaging patients to surgery providing a significant improvement in survival. Even in patients who were not rescued to surgery, survival appeared to be superior in TARE group.

\section{Word count: $250(\max 250)$}

Keywords: Hepatocellular carcinoma; Portal vein tumor thrombosis; Sorafenib; Yttrium-90 microspheres; Trans-arterial radioembolization

\section{Introduction}

Hepatocellular carcinoma (HCC) is the most frequent primary malignant liver tumor, and it represents the fourth leading cause of cancer-related death.(1,2) Despite recommended surveillance in at-risk patients, a high number of tumors are recognized at an advanced stage, when cure is no longer possible.(3) Portal vein tumor thrombosis (PVTT) develops in more than $30 \%$ of HCC patients during the disease course being a parameter of aggressiveness and a strong negative prognostic factor,(4-7) ranking the disease at an advanced stage conforming to the Barcelona Clinic Liver Cancer classification (BCLC).(2)

Sorafenib, an oral multikinase inhibitor, is the worldwide recommended therapy for advanced HCC. $(2,8)$ Systemic treatment with sorafenib was found to stabilize disease progression by its action on cell growth.(9) Moreover, sorafenib extend life expectancy but is not effective in shrinking tumors and a decrease in dose or interruption are often needed for adverse effects.(10-12)

Transarterial radioembolization (TARE) is a loco-regional technique which consists in the infusion, through the hepatic artery, of microspheres carrying an yttrium-90 isotope, which deliver high energy of beta-radiation into the tumor. TARE has been reported to induce a significant decrease in tumor size with a low-grade toxicity, prolonging time to progression and survival.(13-15) TARE can be used with safety in patients with portal vein thrombosis (PVT) for its low risk of liver ischemia(16,17) 
and it has been successfully performed in downstaging strategy in liver transplantation or resective surgery setting.(18-20)

Recently, two randomized trials, SARAH and SIRveNIB, did not show better survival of TARE over sorafenib in patients with advanced HCC. $(11,21)$ The failure could be partially explained by the inclusion of a large share of patients with PVTT extended to the major portal trunk in the TARE arm, since it has recently been reported that the extension of PVTT to the main portal trunk makes TARE treatment futile.(22) Therefore, the scope of our work was to retrospectively investigated the outcome of patients treated with TARE or sorafenib as first-line therapy, focusing on patients with HCC and PVTT limited to the intra-hepatic branches.

\section{Patients and methods}

\section{Patients}

This retrospective study consisted of consecutive HCC patients treated with TARE or sorafenib between 2012 and 2018 at five large hospitals in Northwestern Italy: University Hospital "Città della Salute e della Scienza” Turin, Mauriziano Hospital Turin, Santa Croce e Carle General Hospital Cuneo, Regional Hospital of Aosta Valley Aosta, University Hospital "Maggiore della Carità" Novara. The treatment protocols were authorized by Institutional Ethical Committees and informed consent was obtained by all patients.

Inclusion criteria were naïve patients with Child-Pugh A liver cirrhosis, Eastern Cooperative Oncology Group Performance Status ECOG 0 or 1 and HCC with neoplastic portal vein thrombosis limited to the intra-hepatic branches. Neoplastic portal vein thrombosis was classified conforming to the Liver Cancer Study Group of Japan (LCSGJ):(23)VP1 (distal to but not in second-order branches of the portal vein), VP2 (in second-order branches of the portal vein), VP3 (in first-order branches of the portal vein), VP4 (tumor thrombus in the main trunk of the portal vein and/or contralateral to the trunk). Exclusion criteria were extra-hepatic localizations, presence of non-neoplastic portal vein 
thrombosis, presence of neoplastic portal vein thrombosis VP4 and any previous and subsequent therapy. Histology or non-invasive imaging criteria were used for the diagnosis of HCC.(24) Differentiation of portal vein thrombosis from tumorous portal vein infiltration was made with great precision by contrast-enhanced imaging techniques.(25-28)

\section{TARE procedure}

All patients underwent a standardized workup before treatment; an angiographic study was performed to assess collateral vessels supplying extrahepatic organs and subsequently $150 \mathrm{MBq}$ of technetium99-labeled macroaggregated albumin (99-Tc-MAA) were infused to evaluate the vascular distribution of the microspheres. A total body planar image and a photon emission computed tomography (SPECT-CT) were acquired. In order to administer the optimal tumoricide dose and not to exceed 30 Gy to the lung and 40 Gy to the surrounding liver parenchyma, activities were determined using the multicompartimental MIRD model. $(29,30)$

Transarterial radioembolization was performed, within two weeks, according to the manufacturer guidelines using either yttrium-90 resin (SIR-Spheres; Sirtex Medical Europe, GmbH) or yttrium-90 glass (TheraSphere, BTG International, London, UK) microspheres.(31)

\section{Sorafenib treatment}

Sorafenib was started at the recommended dose of $400 \mathrm{mg}$ twice a day, with dose modifications or interruption according to adverse effects (AEs), graded in agreement with the National Cancer Institute Common Toxicity Criteria (CTCAE3.0).

\section{Follow up and response}

Liver function, serum $\alpha$-fetoprotein (AFP) and physical examinations were performed every month for the first 6 months and then every 3 months. Contrast enhanced computed tomography (CT) was performed at second month after treatment and every 3 or 6 months according to the clinical course 
of the disease. Treatment response was evaluated according to the response evaluation criteria in solid tumors (mRECIST) and the EASL criteria on vascular enhancement. $(32,33)$

\section{Statistical analysis}

Continuous variables were presented as median and interquartile range (IQR); categorical variables were expressed with number and percentage. TARE patients were compared with sorafenib using Mann-Whitney or Fisher's exact tests, as appropriate. The follow-up time was considered from the start of therapy with TARE or sorafenib until the date of death or last visit. The follow-up time was also censored at December 2019. Kaplan-Meier survival estimate were used to evaluate the overall survival in the propensity score analysis, the significance was assessed using Log-rank test. The effect of the outcome was expressed as Hazard Ratio (HR), Schoenfeld test was performed for test the proportional hazard assumption. P-values below 0.05 were considered statistically significant. To adjust for potential patient selection bias, attributable to non-randomized assignment of treatment, a propensity score analysis matching (PSM) and weighting (IPTW) was applied. Four independent prognostic factors were considered in the model: PVTT type 3, number of lesions, alpha-fetoprotein levels and disease progression time. Since both PSM and IPTW do not consider the best model of variable selection and residual imbalance between groups due to the small number of patients, a further analysis based on Bayesian Model Averaging (BMA) was performed on all patients. Hazard Ratio (HR) and the probability of a significant result provided the measure of the size of the association(34). (34). Probabilities greater than 95\% were considered an indication of strong association, probabilities between 75 and $95 \%$ a positive indication of association and probabilities smaller than $75 \%$ were considered a weak evidence of an effect.(35) R version 3.6.1 was used to perform all analyses.(36) 


\section{Results}

Overall, 65 out of 286 patients with HCC and PVTT were recruited in the study. Forty-one were treated with TARE and 24 with sorafenib as first-line treatment. Twenty-three were treated with yttrium-90 resin microspheres and 18 with yttrium-90 glass microspheres. Several patients treated with sorafenib were excluded from the analysis due to previous treatments or due to the invasion of the PVTT in the main trunk.

Eleven patients were rescued to surgery: three patients underwent orthotopic liver transplantation (OLT) and 8 major right hepatectomy. Ten patients obtained a downstaging with TARE and 1 with sorafenib. Eight patients (73\%) were still alive at the end of follow-up. Mean survival of the downstaging group was 54 months. Because of the small sample size of downstaged patients, no statistical inferential analysis was performed in this group.

Baseline demographic properties of the patients are listed in Table 1. Gender male predominance and higher MELD score in the sorafenib group were the only significant differences.

Median overall survival was 20.3 months (95\% CI: 10.8-50.0) versus 9.1 months (95\% CI: 5.8-18.5) in TARE versus sorafenib patients, respectively $(\mathrm{p}=0.001)$.

The 1, 2 and 3 -year OS rates were $64.5 \%, 42.6 \%$ and $37.3 \%$ vs $39.1 \%, 13.0 \%$ and $0 \%$ in TARE and sorafenib patients, respectively (Figure 1). At Univariate Cox model patients undergoing TARE had a longer survival in comparison to patients receiving sorafenib (HR 0.359; 95\% CI:0.190-0.678; $\mathrm{p}=0.002)$.

The propensity matched score analysis (23 TARE vs 23 sorafenib patients) confirmed a median survival of 22.4 months (95\% CI 10.8-NA) in the TARE group and 9.1 months (95\% CI 5.8-18.5) in the sorafenib group $(\mathrm{p}=0.002)$. The 1, 2 and 3- year OS rates in TARE and sorafenib group were $65.2 \%, 43.1 \%$ and $34.5 \%$ vs $39.1 \%, 13 \%$ and $0 \%$ respectively (Figure 2). Univariate Cox model confirmed the effect of the TARE on OS (HR 0.341; 95\% CI:0.168-0.691; $\mathrm{p}=0.003$ ).

At propensity score weighting, performed on all treated patients ( $n=31$ TARE vs $n=23$ sorafenib), overall median survival resulted 20.3 months (95\% CI: 10.8-50.0) in TARE and 10.5 months (95\% 
CI: 5.7-19.4) in sorafenib group $(\mathrm{p}=0.003)$. The 1,2 and 3-year OS rates in TARE and sorafenib group were $64.5 \%, 42.6 \%$ and $37.3 \%$ vs $40.5 \%, 16.4 \%$ and $0 \%$ respectively (Figure 3 ). Univariate Cox model confirmed the effect of the TARE on OS (HR 0.381; 95\% CI:0.201-0.720; p=0.003) (Table 2).

An additional analysis using Bayesian Model Averaging (BMA) was conducted on the entire cohort. As shown in Table 3 only TARE showed a strong correlation with the survival (HR 0.43 with a probability of being < 1 , i.e. protective effect, equal to 0.976 with $95 \%$ credible interval: $0,19-0,99$ ). 


\section{Discussion}

The clinical value of trans-arterial radioembolization with yttrium-90 microspheres in the management of advanced hepatocellular carcinoma is a matter for debate. Growing evidence suggest the role of radioembolization as a safe and efficient treatment for unresectable HCC. $(17,37,38)$ Nevertheless, for many years randomized controlled trials have not been conducted, probably due to the intrinsic complexity of the procedure, requiring a multidisciplinary skilled team, that limits the spread of radioembolization.

Our retrospective multicenter study, focused on a highly selected cohort of patients with locally advanced hepatocellular carcinoma and PVTT limited to the intra-hepatic branches, showed that radioembolization was more performing than sorafenib in terms of survival and downstaging to further surgery intervention.

The 1, 2 and 3-year OS rates in TARE and sorafenib group were $64.5 \%, 42.6 \%$ and $37.3 \%$ vs $39.1 \%$, $13.0 \%$ and $0 \%$, respectively and overall median survival time was 2 time higher in TARE compared to sorafenib patients (20.3 months vs 9.1). A significant improvement of survival in TARE group (HR 0.359; 95\% CI:0.190 0.678, p=0.002) was demonstrate in all survival analysis.

Recently, two randomized controlled trials, the "Selective Internal Radiation Therapy Versus Sorafenib" (SIRveNIB) trial(21) and the "SorAfenib versus Radioembolization in Advanced Hepatocellular carcinoma" (SARAH)(11), failed to demonstrate the superiority of radioembolization versus sorafenib. Similar median overall survival was observed in both groups: 8.8 months versus 10 months in the SIRveNIB trial and 8.0 months versus 9.9 months in the SARAH trial for TARE and sorafenib, respectively. In our opinion, the inclusion of a significant proportion of patients with neoplastic invasion of the main portal trunk in these two trials may have adversely affected the outcome of the patients treated with TARE. Moreover, a recent study shows that PVTT extension, bilirubin level and tumor burden have an independent effect on long-term survival of TARE, while the extension of the PVTT to the main trunk makes futile the treatment with radioembolization. $(22,39)$ 
Furthermore, our study showed that TARE resulted more performing than sorafenib in downsizing/downstaging patients to a surgical curative-intent treatment: 10 of 41 patients were rescued to surgery with TARE, while only 1 of 24 with sorafenib. $73 \%$ (8/11 patients) were still alive at the end of the follow-up and the mean survival time was 54 months. It is important to underline that, at baseline, patients rescued to surgery have a lower AFP level, a lower number of lesions and a lower extension of PVTT compared to the others. These data should be taken in account when consider a treatment for patient with advanced HCC but have to be validated by more extensive studies.

Main significant biases of our study were the low number of enrolled patients and the retrospective collection of the data. Due to the restricted inclusion criteria many patients treated with sorafenib were excluded from the analysis, however the two groups of treatment at baseline were demographically and clinically similar. To overcome these limitations, we used the propensity score method to evaluate the impact of radioembolization and sorafenib on overall survival and Bayesian analysis to analyze the effect of different variables on the outcome. Both propensity score matched analysis and propensity score weighted analysis concurred to show an improvement in overall survival in TARE group in comparison to systemic treatment. These data indicate TARE as the best performing treatment in selected patients (HR 0.341; 95\% CI:0.168-0.691; $\mathrm{p}=0.003$ and HR 0.381; 95\% CI:0.201-0.720; p=0.003 respectively). Similarly, Bayesian Model Averaging (BMA) analysis demonstrated that patients treated with TARE had a higher overall survival than sorafenib (HR 0.43 with $95 \%$ credible interval: $0,19-0,99)$, with a high probability of significant association and high probability of inclusion $(90.7 \%)$.

Another possible limitation of our study is related to the adoption of two different devices for delivering selective internal radiation to the tumor; in fact, the two microsphere products are slightly different when it comes to number of particles, density, size and method of infusion. 
However, it must be considered that in literature the survival and radiological response reported in HCC patients are similar for the two techniques. $(39,40)$ In our opinion, we believe that the outcome is determined more by the accurate dosimetric study than by the device used.

In Advanced HCC patients the choice of the correct therapy is always a big challenge and many aspects have to be considered such as quality of life, costs and local availability. However, even if in this study we did not analyzed these data, a recent study compared cost of using radioembolization versus sorafenib and lenvatinib as primary treatment from the healthcare payer perspectives in Italy, France, Spain and UK, given the improved quality of life and the potential to rescue patients to curative therapies.(41) TARE resulted in cost reduction in all four states, even including the costs of curative treatment. Other studies were published in the past years supporting the same results.(4244)

The therapeutic horizon of HCC is rapidly evolving and different immune-oncotherapeutic drugs, alone or in combination, recently showed encouraging results with regards to oncological response and therefore of downstaging capability to rescue to curative treatments.(45-48)

The widespread adoption of these upcoming treatments would inevitably lead to an increase in the total oncologic cost, that could be difficult to bear for the healthcare system.

In conclusion, our study showed that TARE can be considered as first option in selected patients with advanced HCC with branches portal vein tumor thrombosis and without extrahepatic disease; however, prospective studies are required to compare radioembolization with new upcoming immune-chemotherapeutic agents.

\section{Acknowledgments}

None to declare 

mortality worldwide for 36 cancers in 185 countries. CA Cancer J Clin. 2018 Nov;68(6):394-424.

2. Galle PR, Forner A, Llovet JM, Mazzaferro V, Piscaglia F, Raoul JL, et al. EASL Clinical Practice Guidelines: Management of hepatocellular carcinoma. J Hepatol. 2018 Jul 1;69(1):182-236.

3. Thomas MB, Jaffe D, Choti MM, Belghiti J, Curley S, Fong Y, et al. Hepatocellular carcinoma: Consensus Recommendations of the National Cancer Institute Clinical Trials Planning Meeting. Vol. 28, Journal of Clinical Oncology. 2010. p. 3994-4005. Llovet JM, Bustamante J, Castells A, Vilana R, Del Carmen Avuso M, Sala M, et al. Natural history of untreated nonsurgical hepatocellular carcinoma: Rationale for the design and evaluation of therapeutic trials. Hepatology. 1999 Jan;29(1):62-7.

5. Schöniger-Hekele M, Müller C, Kutilek M, Oesterreicher C, Ferenci P, Gangl A. Hepatocellular carcinoma in Central Europe: Prognostic features and survival. Gut. 2001 Jan;48(1):103-9.

6. Cabibbo G, Enea M, Attanasio M, Bruix J, Craxí A, Camma C. A meta-analysis of survival rates of untreated patients in randomized clinical trials of hepatocellular carcinoma. Hepatology. 2010 Apr;51(4):1274-83. Carr BI, Guerra V. A Hepatocellular Carcinoma Aggressiveness Index and Its Relationship to Liver Enzyme Levels. Oncol. 2016 Apr $1 ; 90(4): 215-20$.

8. Heimbach JK, Kulik LM, Finn RS, Sirlin CB, Abecassis MM, Roberts LR, et al. AASLD guidelines for the treatment of hepatocellular carcinoma. Hepatology. 2018 Jan 1;67(1):358-80.

9. Chaparro M, González Moreno L, Trapero-Marugán M, Medina J, Moreno-Otero R. Review article: Pharmacological therapy for hepatocellular carcinoma with sorafenib and other oral agents. Vol. 28, Alimentary Pharmacology and Therapeutics. 2008. p. $1269-77$. Pinter M, Sieghart W, Graziadei I, Vogel W, Maieron A, Königsberg R, et al. Sorafenib in Unresectable Hepatocellular Carcinoma from Mild to Advanced Stage Liver Cirrhosis. Oncologist. 2009 Jan;14(1):70-6.

11. Vilgrain V, Pereira H, Assenat E, Guiu B, Ilonca AD, Pageaux GP, et al. Efficacy and safety of selective internal radiotherapy with yttrium90 resin microspheres compared with sorafenib in locally advanced and inoperable hepatocellular carcinoma (SARAH): an open-label randomised controlled phase 3 trial. Lancet Oncol. 2017 Dec 1;18(12):1624-36. Cheng AL, Kang YK, Chen Z, Tsao CJ, Qin S, Kim JS, et al. Efficacy and safety of sorafenib in patients in the Asia-Pacific region with advanced hepatocellular carcinoma: a phase III randomised, double-blind, placebo-controlled trial. Lancet Oncol. 2009 Jan;10(1):25-34.

13. Díaz-González Á, Reig M, Bruix J. Treatment of Hepatocellular Carcinoma. Dig Dis. 2016 Jun 1;34(5):597-602.

14. Toso C, Mentha G, Kneteman NM, Majno P. The place of downstaging for hepatocellular carcinoma. Vol. 52, Journal of Hepatology. 2010. p. $930-6$.

15. Salem R, Lewandowski RJ, Mulcahy MF, Riaz A, Ryu RK, Ibrahim S, et al. Radioembolization for Hepatocellular Carcinoma Using Yttrium-90 Microspheres: A Comprehensive Report of Long-term Outcomes. Gastroenterology. 2010;138(1):52-64.

16. Sangro B, Iñarrairaegui M, Bilbao JI. Radioembolization for hepatocellular carcinoma. Vol. 56, Journal of Hepatology. 2012. p. 464-73.

17. Mazzaferro V, Sposito C, Bhoori S, Romito R, Chiesa C, Morosi C, et al. Yttrium-90 radioembolization for intermediate-advanced hepatocellular carcinoma: A phase 2 study. Hepatology. 2013 May;57(5):1826-37.

18. Salem R, Gordon AC, Mouli S, Hickey R, Kallini J, Gabr A, et al. Y90 Radioembolization Significantly Prolongs Time to Progression Compared With Chemoembolization in Patients With Hepatocellular Carcinoma. Gastroenterology. 2016 Dec 1;151(6):1155-1163.e2.

19. Edeline J, Lenoir L, Boudjema K, Rolland Y, Boulic A, Le Du F, et al. Volumetric changes after 90Y radioembolization for hepatocellular carcinoma in cirrhosis: An option to portal vein embolization in a preoperative setting? Ann Surg Oncol. 2013 Aug;20(8):2518-25. 
21. Chow PKH, Gandhi M, Tan SB, Khin MW, Khasbazar A, Ong J, et al. SIRveNIB: Selective internal radiation therapy versus sorafenib in Asia-Pacific patients with hepatocellular carcinoma. J Clin Oncol. 2018 Jul 1;36(19):1913-21. Spreafico C, Sposito C, Vaiani M, Cascella T, Bhoori S, Morosi C, et al. Development of a prognostic score to predict response to Yttrium90 radioembolization for hepatocellular carcinoma with portal vein invasion. J Hepatol. 2018 Apr 1;68(4):724-32. Kudo M, Izumi N, Kubo S, Kokudo N, Sakamoto M, Shiina S, et al. Report of the 20th Nationwide follow-up survey of primary liver cancer in Japan. Hepatol Res. 2020 Jan 1;50(1):15-46. Bruix J, Sherman M. AASLD PRACTICE GUIDELINE Management of Hepatocellular Carcinoma. 2005; Piscaglia F, Gianstefani A, Ravaioli M, Golfieri R, Cappelli A, Giampalma E, et al. Criteria for diagnosing benign portal vein thrombosis in the assessment of patients with cirrhosis and hepatocellular carcinoma for liver transplantation. Liver Transplant. 2010 May;16(5):658-67. Rossi S, Ghittoni G, Ravetta V, Torello Viera F, Rosa L, Serassi M, et al. Contrast-enhanced ultrasonography and spiral computed tomography in the detection and characterization of portal vein thrombosis complicating hepatocellular carcinoma. Eur Radiol. 2008 Aug $28 ; 18(8): 1749-56$. Sorrentino P, Tarantino L, D’Angelo S, Terracciano L, Ferbo U, Bracigliano A, et al. Validation of an extension of the international noninvasive criteria for the diagnosis of hepatocellular carcinoma to the characterization of macroscopic portal vein thrombosis. J Gastroenterol Hepatol. 2011 Apr 1;26(4):669-77. Catalano OA, Choy G, Zhu A, Hahn PF, Sahani D V. Differentiation of malignant thrombus from bland thrombus of the portal vein in patients with hepatocellular carcinoma: Application of diffusion-weighted MR imaging. Radiology. 2010 Jan 14;254(1):154-62. Gulec SA, Mesoloras G, Stabin M. Dosimetric techniques in 90Y-microsphere therapy of liver cancer: The MIRD equations for dose calculations. J Nucl Med. 2006 Jul;47(7):1209-11. Ho S, Lau WY, Leung TWT, Chan M, Ngar YK, Johnson PJ, et al. Partition model for estimating radiation doses from yttrium-90 microspheres in treating hepatic tumours. Eur J Nucl Med. 1996;23(8):947-52. Kennedy A, Nag S, Salem R, Murthy R, McEwan AJ, Nutting C, et al. Recommendations for Radioembolization of Hepatic Malignancies Using Yttrium-90 Microsphere Brachytherapy: A Consensus Panel Report from the Radioembolization Brachytherapy Oncology Consortium. Int J Radiat Oncol Biol Phys. 2007 May 1;68(1):13-23. Lencioni R, Llovet JM. Modified recist (mRECIST) assessment for hepatocellular carcinoma. Vol. 30, Seminars in Liver Disease. 2010. p. $52-60$. Bruix J, Sherman M, Llovet JM, Beaugrand M, Lencioni R, Burroughs AK, et al. Clinical management of hepatocellular carcinoma. Conclusions of the Barcelona-2000 EASL conference. European Association for the Study of the Liver. J Hepatol. 2001 Sep;35(3):421-30. Volinsky CT, Madigan D, Raftery AE, Kronmal RA. Bayesian Model Averaging in Proportional Hazard Models: Assessing the Risk of a Stroke. J R Stat Soc Ser C (Applied Stat. 1997 Jan 6;46(4):433-48. Kass RE, Raftery AE. Bayes factors. J Am Stat Assoc. 1995;90(430):773-95. Team r core. A Language and Environment for Statistical Computing. Vienna, Austria: R Foundation for Statistical Computing; 2019. Sangro B, Carpanese L, Cianni R, Golfieri R, Gasparini D, Ezziddin S, et al. Survival after yttrium-90 resin microsphere radioembolization of hepatocellular carcinoma across Barcelona clinic liver cancer stages: A European evaluation. Hepatology. 2011 Sep 2;54(3):868-78. Iarrairaegui M, Thurston KG, Bilbao JI, D’Avola D, Rodriguez M, Arbizu J, et al. Radioembolization with use of yttrium-90 resin microspheres in patients with hepatocellular carcinoma and portal vein thrombosis. J Vasc Interv Radiol. 2010 Aug 1;21(8):1205-12. Cardarelli-Leite L, Chung J, Klass D, Marquez V, Chou F, Ho S, et al. Ablative Transarterial Radioembolization Improves Survival in Patients with HCC and Portal Vein Tumor Thrombus. Cardiovasc Intervent Radiol. 2020 Mar 1;43(3):411-22. 
raDioembolization: Comparing survival in unresectable hepatocellular carcinoma using pretreatment partition model dosimetry. $\mathrm{J}$ Nucl Med. 2017 Aug 1;58(8):1334-40.

41. Pollock R, Colaone F, Guardiola L, Shergill S, Brennan VK. A cost analysis of SIR-Spheres yttrium-90 resin microspheres versus tyrosine kinase inhibitors in the treatment of unresectable hepatocellular carcinoma in France, Italy, Spain and the UK. J Med Econ. 2020 Feb 18;1.

42. Rognoni C, Ciani O, Sommariva S, Bargellini I, Bhoori S, Cioni R, et al. Trans-arterial radioembolization for intermediate-advanced hepatocellular carcinoma: A budget impact analysis. BMC Cancer. 2018 Jul 5;18(1):715.

43. Rognoni C, Ciani O, Sommariva S, Tarricone R. Real-World Data for the Evaluation of Transarterial Radioembolization versus Sorafenib in Hepatocellular Carcinoma: A Cost-Effectiveness Analysis. Value Heal. 2017 Mar 1;20(3):336-44.

44. Lucà MG, Nani R, Schranz M, De Giorgio M, Iegri C, Agazzi R, et al. Treatment of hepatocellular carcinoma: A cost analysis of yttrium-90 transarterial radioembolization versus sorafenib. Futur Oncol. 2018 Apr 1;14(8):727-35.

45. Finn RS, Qin S, Ikeda M, Galle PR, Ducreux M, Kim TY, et al. Atezolizumab plus bevacizumab in unresectable hepatocellular carcinoma. N Engl J Med. 2020 May 14;382(20):1894-905.

46. Schwacha-Eipper B, Minciuna I, Banz V, Dufour JF. Immunotherapy as a downstaging therapy for liver transplantation. Hepatology. 2020 Mar 14;hep.31234.

47. Chen X, Zhang Y, Zhang N, Ge Y, Jia W. <p>Lenvatinib combined nivolumab injection followed by extended right hepatectomy is a feasible treatment for patients with massive hepatocellular carcinoma: a case report</p>. Onco Targets Ther. 2019 Sep 9;Volume 12:73559.

48. Zhang T, Zhang L, Xu Y, Lu X, Zhao H, Yang H, et al. Neoadjuvant therapy and immunotherapy strategies for hepatocellular carcinoma. Am J Cancer Res. 2020;10(6):1658-67. 


\section{TABLES}

Table 1. Baseline characteristics of patients by treatment

\begin{tabular}{|l|c|c|c|c|}
\hline & Sorafenib & TARE & $\begin{array}{c}\text { p- } \\
\text { value }\end{array}$ & Downstaging \\
\hline Number of patients & 23 & 31 & 11 \\
\hline Age (years) & $75.00[62.00,81.00]$ & $73.00[63.00,82.00]$ & 0.800 & $77.00[68.50,80.00]$ \\
\hline Male sex, n (\%) & $23(100.0)$ & $25(80.6)$ & 0.032 & $11(100.0)$ \\
\hline $\begin{array}{l}\text { Etiology, n (\%) } \\
\text { Viral }\end{array}$ & $13(56.5)$ & $21(67.7)$ & 0.569 & $8(72.7)$ \\
\hline INR & $1.07[1.00,1.30]$ & $1.09[1.00,1.13]$ & 0.218 & $1.07[1.00,1.16]$ \\
\hline Total bilirubin (mg/dl) & $1.10[0.70,1.31]$ & $0.90[0.70,1.05]$ & 0.131 & $1.00[0.85,1.30]$ \\
\hline Albumin (g/dl) & $38.00[36.00,40.00]$ & $36.00[35.00,39.50]$ & 0.280 & $36.00[35.00,38.50]$ \\
\hline Creatinine (mg/d) & $0.80[0.68,0.97]$ & $0.80[0.65,0.90]$ & 0.438 & $0.80[0.66,0.90]$ \\
\hline MELD score & $9.00[8.00,10.00]$ & $8.00[7.00,9.00]$ & 0.035 & $9.00[8.00,9.50]$ \\
\hline $\begin{array}{l}\text { Main tumor diameter } \\
\text { (mm) }\end{array}$ & $48.00[40.00,70.00]$ & $55.00[36.50,72.50]$ & 0.955 & $70.00[51.50,80.00]$ \\
\hline $\begin{array}{l}\text { Number of lesions, n (\%) } \\
\text { Unifocal }\end{array}$ & $7(30.4)$ & $10(32.3)$ & 1.000 & $4(36.4)$ \\
\hline PVTT Type 3, n (\%) & $15(65.2)$ & $14(45.2)$ & 0.175 & $1(9.1)$ \\
\hline AFP (ng/ml) & $13(56.5)$ & $14(45.2)$ & 0.416 & $24.00[9.00,343.50]$ \\
\hline AFP $\geq$ 400 (ng/ml), n (\%) & $907.00[39.20,2028.00]$ & $326.00[10.55,1279.00]$ & 0.583 & $3(27.3)$ \\
\hline
\end{tabular}

MELD, Model for End-stage Liver Disease; INR, International Normalized Ratio, AFP, Alpha-fetoprotein.

${ }^{1}$ Comparison between patients treated with TARE and patients treated with sorafenib

Table 2. TARE vs sorafenib Hazard ratio

\begin{tabular}{|l|c|c|c|}
\hline & Hazard Ratio & Confidence interval 95\% & p-value \\
\hline No propensity score & 0.359 & $0.190-0.678$ & $\mathrm{p}=0.002$ \\
\hline Propensity score matched & 0.341 & $0.168-0.691$ & $\mathrm{p}=0.003$ \\
\hline Propensity score weighted & 0.381 & $0.201-0.720$ & $\mathrm{p}=0.003$ \\
\hline
\end{tabular}

Table 3. Bayesian model averaging results.

\begin{tabular}{|l|l|l|l|l|l|}
\hline \multicolumn{1}{|c|}{ Variable } & \multicolumn{1}{|c|}{$\begin{array}{c}\text { Probability of } \\
\text { significant association } \\
\text { with survival }\end{array}$} & $\begin{array}{c}\text { Probability of } \\
\text { inclusion }\end{array}$ & \multicolumn{2}{c|}{ 95\% Credible Interval } \\
\hline AFP = < 400 & 0.87 & 0.690 & 29.50 & 0.50 & 1.48 \\
\hline PVTT, Type 3 & 0.94 & 0.625 & 17.50 & 0.66 & 1.35 \\
\hline $\begin{array}{l}\text { Number of } \\
\text { lesions }>\text { 1 }\end{array}$ & 1.02 & 0.568 & 11.20 & 0.79 & 1.32 \\
\hline Progression & 1.68 & 0.850 & 63.70 & 0.63 & 4.52 \\
\hline TARE & 0.43 & 0.976 & 90.70 & 0.19 & 0.99 \\
\hline
\end{tabular}


Figure 1: Kaplan-Meier without Propensity Score

Figure 2: Kaplan-Meier after Propensity Score Matching

Figure 3: Kaplan-Meier after Propensity Score Weighting 
